\title{
Morbidades referidas por trabalhadoras que produzem joias folheadas em Limeira, SP*
}

\author{
Morbidity reported by workers who produce plated jewelry in \\ Limeira, SP, Brazil
}

\begin{abstract}
1 Professora adjunta do Departamento de Psicologia da Universidade Federal de Mato Grosso do Sul (UFMS), Campus do Pantanal, Corumbá, MS, Brasil.
\end{abstract}

*Artigo baseado na tese de doutorado em Saúde Coletiva de Vanessa Catherina Neumann Figueiredo, intitulado Trabalho e gênero: condições de saúde das mulheres no setor de bijuterias e folheados, defendida em 2008 na Faculdade de

Ciências Médicas da Unicamp, Campinas, SP, Brasil.

Trabalho apresentado por Vanessa Catherina Neumann Figueiredo, com o título Morbidades referidas pelas trabaIhadoras do setor de bijuterias e folheados em Limeira, no VIII Congresso Mundial de Epidemiologia - Porto Alegre, 22 de set. 2008.

Financiado parcialmente com bolsa da CAPES.

\section{Contato:}

Vanessa Catherina Neumann

Figueiredo

Departamento de Psicologia-Campus do Pantanal

Avenida Rio Branco, 1.270 - Universitário - Corumbá - MS

CEP: 79.304-902

Caixa postal: 252

E-mail:

vanessa_figueiredo@hotmail.com

\section{Resumo}

Introdução: a cidade de Limeira (SP) se caracteriza por ser um polo de empresas do ramo de joias folheadas e bijuterias, sendo a maior parte da mão de obra composta por mulheres. Objetivo: verificar as morbidades referidas por mulheres expostas ao cianeto no setor de bijuterias e joias folheadas na cidade de Limeira em 2007. Metodologia: estudo transversal realizado com 383 trabalhadoras, comparando-se um grupo de 191 mulheres trabalhadoras em galvanoplastias consideradas qualitativamente expostas ao cianeto com um grupo de 192 mulheres não expostas ao cianeto. Foi aplicado o Índice de Capacidade para o Trabalho, o Questionário de Tolerância de Fagerström e um questionário sociodemográfico e ocupacional. Resultados: O grupo considerado exposto ao cianeto apresentou: menor média de tempo de trabalho no cargo ( $\mathrm{p}=0,008)$, maior proporção que trabalha com produtos químicos ( $p<0,001)$, que usa Equipamento de Proteção Individual ( $p<0,001)$, que recebe equipamento das empresas em que trabalham $(\mathrm{p}<0,001)$, menor quantidade de morbidades referidas $(p=0,042)$, mas maior prevalência de bronquite crônica $(p=0,027)$, sinusite crônica $(\mathrm{p}=0,022)$ e disfunções da tireoide $(\mathrm{p}=0,022)$. Conclusões: os resultados apontam para a necessidade de pesquisas de coorte que analisem a associação entre os agravos apresentados e a exposição ao cianeto.

Palavras-chave: saúde; trabalho; cianeto, galvanoplastia.

\begin{abstract}
Introduction: The city of Limeira in São Paulo, Brazil, is characterized by a cluster of companies producing jewelry, plated jewelry, and fashion jewelry, with most of the laborforce composed of women. Objective: The purpose of this research was to assess the health conditions of workers from gold plated and costume jewelry manufacturers in Limeira, in 2007. Methodology: Transversal study involving 383 women, comparing a group of 191 workers of electroplating sites considered exposed to cyanide to a group of 192 non-exposed. Work Index Capacity, Fagerström Scale of Tolerance, and a socio-demographic and occupational questionnaire were applied. Results: The exposed group presented shorter average of time working in the company ( $p=0.008$ ), higher proportion of workers dealing with chemicals ( $p$ $<0.001)$, wearing Personal Protective Equipment (PPE) ( $p<0.001)$, and receiving PPE provided by the companies where they worked ( $p<0.001)$. The group exposed to cyanide also presented lower proportion in reported morbidity ( $p=0.042)$, but higher prevalence of chronic bronchitis $(p=0.027)$, chronic sinusitis $(p=0.022)$, and thyroid disorders ( $p=0.022$ ). Conclusion: The results indicate the need for cohort studies to examine the association between the reported diseases and cyanide exposure.
\end{abstract}

Keywords: health; work; cyanide; electroplating. 


\section{Introdução}

A morbidade é uma das informações essenciais para conhecer as condições de saúde de uma população, pois possibilita planejar ações e medidas preventivas em seu benefício (MELO; CAMPINAS, 2010). Apesar do seu caráter subjetivo, a morbidade autorreferida tem sido incorporada em investigações epidemiológicas por meio da aplicação de entrevista domiciliar, questionário ou inquérito populacional.

Com o propósito avaliar o estado de saúde da população, a morbidade registra o número de pessoas incapacitadas ou funcionalmente doentes que usam os diferentes tipos de serviços de saúde (CESAR et al., 1996). Já a morbidade reprimida - aquela ainda não registrada devido à falta de uso ou de acesso aos serviços de saúde pela população - representa uma das estratégias que podem avaliar a situação de saúde dos trabalhadores e a determinação social da saúde-doença (LEBRÃO; DUARTE, 2003), pois revela as desigualdades existentes entre os grupos no que tange às relações de gênero, etnia, cultura e trabalho, bem como suas necessidades específicas de saúde (PINHEIRO et al., 2002). Contudo, mesmo sendo considerada um indicador importante para a escolha das ações saneadoras adequadas, ainda são raros os trabalhos voltados para a análise da morbidade referida por mulheres trabalhadoras no Brasil (GOMES; TANAKA, 2003), grupo focado no nosso estudo.

De acordo com Suzigan et al. (2001), a cidade de Limeira apresenta uma crescente concentração de empresas na industria de joias, bijuterias e folheados, sendo que, já em 2000, estimativas locais computavam a presença de 15 a 20 mil postos de trabalho e, em 2005, dados extraoficiais apontavam aproximadamente 35 mil pessoas trabalhando nas fábricas de joias na região, sendo a maior parte da mão de obra composta por mulheres.

Praticamente toda a cadeia produtiva está instalada nesse município, que conta com fabricantes de joias folheadas, peças brutas, máquinas e equipamentos, prestadores de serviço para o setor (como galvanoplastia, montagem, solda, usinagem e outros), distribuidora de insumos químicos para os banhos galvânicos, bem como os diversos canais de comercialização existentes na região, tanto para o mercado nacional, como internacional (SAMPAIO, 2002).

A produção de bijuterias e folheados envolve uma série de processos industriais, distribuídos entre a fabricação e a montagem de peças brutas e o processo de tratamento de superfícies (galvanoplastia). Na fabricação das peças brutas (as quais ainda não passaram pelo banho de ouro ou prata), são usados processos metalúrgicos, principalmente a fundição, além dos processos de cera perdida, baixa fusão, fotocorrosão, estamparia e rebarbação (SANTOS; YAMANAKA; PACHECO, 2005). Tais processos exigem um trabalho repetitivo e sustentado, com o braço em abdução e flexão, por vezes, o uso prolongado de martelo, com supinação repetida e pronação do antebraço, extensão e flexão forte de pulso, ou garra com um braço estendido ou cotovelo completamente dobrado. Já durante a montagem das peças, são muito usados os dedos polegar e indicador e movimento repetitivo do pulso.

Depois de fabricadas e montadas, as peças brutas passam pelo processo de tratamento de superfície, ou galvanoplastia, definido como a tecnologia responsável pela transferência de íons metálicos de uma dada superfície sólida ou meio líquido, denominado eletrólito, para outra superfície, seja ela metálica ou não (FERNANDES, 2005; CRUZ, 2001). As empresas que fazem o tratamento de superfície banham as peças em tanques, onde são usados compostos como cobre alcalino, cobre ácido, níquel, ouro, prata e ródio, sendo nítidos os riscos existentes na exposição a névoas ácidas e vapores contendo contaminantes metálicos e cianetos (SILVA, 1998).

Com isso, além da organização do trabalho que dita ritmos intensos de trabalho, também a exposição a substâncias químicas, em especial ao cianeto, deve ser levada em conta na caracterização do trabalho estudado. Em várias etapas do tratamento de superfície, são usados os cianetos de potássio, de sódio, de cobre e/ou de prata, os quais são rapidamente absorvidos pelo corpo por meio da inalação, da pele, dos olhos e da ingestão, sendo a absorção pulmonar a mais eficiente (AGENCY FOR TOXIC SUBSTANCES AND DISEASE REGISTRY, 2006).

Relatos de galvanoplastas expostos por longo tempo incluem mudanças funcionais na audição, perda de apetite, cefaleia, fraqueza, náusea, tontura, irritação no trato respiratório superior, causando tosse e dificuldade respiratória (CURRY, 1992), além de dermatites (LEWIS, 1996). Após uma intoxicação aguda, podem ocorrer sequelas neurológicas, que resultam em lesão motora permanente - como paralisia e uma síndrome parecida com a parkinsoniana, com prejuízo mental permanente (DI FILIPPO et al., 2008; PENTORE; VENNERI; NICHELLI, 1996; ZHANG et al., 2009). O uso de sais de cianeto por trabalhadores que realizam tratamento de metais aquecidos pode resultar em aumento e/ou disfunção das glândulas tireoidianas, como o hipotireoidismo, em função de falhas em eliminar a substância (QUIROGA; OLMOS, 2009).

Por ser um produto altamente tóxico, a pequena quantidade de $180 \mathrm{mg}$ ou mesmo a inalação de concentrações de ar de 200 a $300 \mathrm{ppm}$ de cianeto podem ser fatais. Em termos de absorção via oral, 
a quantidade de $100 \mathrm{mg}$ de cianeto de sódio pode ser letal (HALL; RUMACK, 1998). A intoxicação aguda pelo cianeto pode gerar vários sintomas e mudanças nos sinais vitais em função da rápida estimulação e seguinte depressão do Sistema Nervoso Central (HALL; RUMACK, 1998), além de poder causar cegueira e danos aos nervos ópticos e à retina (GRANT, 1974).

O cianeto absorvido pode ser eliminado através do pulmão, da saliva ou da urina, mas um dos maiores mecanismos para remover o cianeto do organismo é sua conversão enzimática para tiocianato. $\mathrm{O}$ cianeto compete com a captação do iodo, motivo pelo qual a exposição ao cianeto pode acarretar em hipotireoidismo, o qual pode se manifestar como desânimo, intolerância ao frio, fala arrastada, pele seca, descamativa e infiltrada (mixedema), edema palpebral, cabelos e unhas secos e quebradiços, palidez cutânea. Nas formas mais avançadas, há redução da capacidade intelectual, apatia e sonolência, diminuição da freqüência cardíaca e é comum ocorrer hipermenorreia, anovulação, diminuição da libido e coma (MONTELIUS, 2001).

Partindo-se, portanto, da hipótese de que as mulheres que trabalham nas empresas com processo de galvanoplastia apresentariam piores condições de saúde em função da possibilidade de desenvolver hipotireoidismo ou disfunções tireoidianas associadas à exposição ao cianeto, este estudo buscou levantar as morbidades autorreferidas pelas trabalhadoras da indústria de bijuterias e joias folheadas, na cidade de Limeira (SP), através da autoavaliação da saúde (FIGUEIREDO, 2008).

\section{Métodos}

Realizou-se um estudo transversal com $383 \mathrm{mu}-$ lheres inseridas na indústria de bijuterias e joias folheadas na cidade de Limeira (SP) no ano de 2007. Comparou-se um grupo de 191 mulheres expostas ao cianeto, ou seja, inseridas em empresas com processo de galvanoplastia, com um grupo de 192 mulheres não expostas ao cianeto, ou seja, que montavam ou produziam as peças brutas. Considerou-se que todas as mulheres que trabalhavam nas empresas com tratamento de superfície estavam expostas ao cianeto em função das diferentes atividades, como a de colocar as peças na gancheira para posterior banho de cianeto de ouro ou de prata, serem realizadas no mesmo ambiente de trabalho ou até na mesma bancada.

De acordo com registro fornecido pelo Sindicato dos Trabalhadores Joalheiros do Estado de São Paulo - Limeira, em 2007, havia 1.113 trabalhadoras com carteira de trabalho registrada no setor, sendo que 315 trabalhavam em empresas com tratamento de superfície e 798 em empresas que produziam ou montavam peças brutas. Todas as empresas que constavam no sindicato foram visitadas pela pesquisadora, acompanhada do presidente do sindicato da região, o que facilitou nossa entrada nas empresas, bem como nosso diálogo com as trabalhadoras e convite a elas. Considerando que a prevalência de disfunções tireoidianas é de $0,5 \%$ a $1 \%$ na população em geral (FREITAS; LIMA, 1999; PONTES et al., 2002), calculou-se uma amostra mínima de 164 mulheres expostas ao cianeto (ou seja, que trabalhavam em ambiente que continha cianeto, necessário para realizar os banhos de ouro e prata) e 164 não expostas ao cianeto (ou seja, que apenas montavam ou fabricavam as peças, não trabalhando em ambientes com cianeto), considerando $\mathrm{p} 1=1 \%$ e p $2=8 \%$, com alfa $=5 \%$ e poder de $80 \%$.

Os critérios de exclusão usados foram não trabalhar no setor e não morar em Limeira. Todas as mulheres que não se enquadravam nos critério de exclusão foram convidadas a participar voluntariamente do estudo.

Foram aplicados os seguintes instrumentos:

Questionário Sociodemográfico e Ocupacional: idade, etnia, estado civil, escolaridade, trabalho doméstico, tempo na empresa, no cargo e no setor, uso de Equipamento de Proteção Individual, tipo de trabalho desenvolvido, exposição a substâncias químicas.

Índice de Capacidade para o Trabalho (ICT): as estimativas de prevalência de doenças foram baseadas na morbidade autorreferida, que apresenta como vantagem a rapidez na obtenção da informação e o baixo custo, viabilizando a sua adoção em grandes populações. Para isso, foi utilizada a quarta questão do ICT: Na sua opinião, quais das lesões por acidentes ou doenças citadas abaixo você possui atualmente? Marque também aquelas que foram confirmadas pelo médico (TUOMI et al., 2005). A presença ou não de cada patologia foi respondida no formato sim ou não, seguindo a ordem estabelecida pelo instrumento e sem que houvesse explicações acerca de cada morbidade. Não houve exame clínico para identificação das patologias ou doenças.

Questionário de Tolerância de Fagerström: usadopara identificar e medir a dependência nicotínica, o questionário é composto de três questões que avaliam o fumo matinal, considerado indicador de síndrome de abstinência, e por três questões que avaliam o consumo de cigarros (HALTY et al., 2002; MARQUES; RIBEIRO, 2003).

As variáveis analisadas foram: características sociodemográficas e familiares; características ocupacionais; quantidade e tipos de lesões e doenças (com diagnóstico médico); grau de dependência do tabaco 
na forma de escore, variando de 0 a 10 pontos (leve de 0 a 4 pontos, moderada de 5 a 7 pontos e alta de 8 a 10 pontos); e as variáveis contínuas tempo de tabagismo em anos e quantidade de cigarros por dia.

Para a realização das análises descritivas e dos testes estatísticos, foi utilizado o programa SPSS para Windows versão 10. As variáveis qualitativas foram resumidas e representadas por frequência absoluta (n) e relativa (\%) e as quantitativas por média (dp), mediana, valores mínimo e máximo. Os grupos de interesse foram comparados quanto às variáveis quantitativas pelo Teste $t$ de Student ou pelo Teste não paramétrico de Mann-Whitney. A presença de associação entre a variável de grupo e as demais variáveis qualitativas foi avaliada pelo Teste do Qui-quadrado ou Teste da Razão de Verossimilhança, caso as suposições básicas do Qui-quadrado não estivessem satisfeitas. Adotou-se o nível de significância de 0,05 (a = 5\%) e níveis descritivos (p) inferiores a esse valor foram considerados significantes.

A pesquisa foi aprovada pelo Comitê de Ética da Faculdade de Ciências Médicas da Universidade Estadual de Campinas (Unicamp) e seguiu as diretrizes e normas regulamentadoras de pesquisas envolvendo seres humanos definidas na Resolução nº 196/96 do Conselho Nacional de Saúde.

\section{Resultados}

Com relação aos aspectos sociodemográficos, na comparação entre os grupos, verificou-se uma tendência do grupo exposto apresentar: maior proporção de mulheres entre 20 e 29 anos ( $p=0,054)$; mais mulheres solteiras e menos mulheres separadas $(49,7 \%$ e $4,2 \%$ respectivamente) do que o grupo não exposto (39,1\% e $10,4 \%-p=0,036)$; mais mulheres que completaram até $3^{a}$ série do Ensino Médio $(71,1 \%)$ e que completaram o Ensino Superior (12,3\%) e menos mulheres que completaram até $8^{\mathrm{a}}$ série do Ensino Fundamental $(12,3 \%)$ em relação ao grupo não exposto $(58,1 \%, 5,4 \%$ e 29,6\% respectivamente $-\mathrm{p}<0,001$ ) (Tabela 1).

Quanto aos aspectos ocupacionais, verificou-se que o grupo considerado exposto ao cianeto apresentou menor média de tempo de trabalho no cargo (3,9 anos) do que o grupo não exposto $(4,9$ anos $-\mathrm{p}=0,008) \mathrm{e}$ maior proporção de mulheres que relataram trabalhar com substâncias químicas $(54,8 \%)$ do que no grupo não exposto ao cianeto $(5,1 \%-p<0,001)$ (Tabela 1). Das 185 mulheres que responderam à questão acerca dos tipos de substâncias químicas que manejam, 97 afirmaram ter contato direto com substâncias químicas, com 42,2\% trabalhando apenas com cianeto e $6,7 \%$ com cianeto e também outros produtos. Nenhum teste estatístico pôde ser aplicado para comparar os grupos quanto à distribuição do tempo de contato com substâncias químicas devido ao grande número de categorias desta variável (Tabela 2).

Tabela 1 Faixa etária, estado civil e escolaridade de trabalhadoras expostas (galvanoplastia) e não expostas (montagem) ao cianeto na indústria de joias folheadas, Limeira, 2007

\begin{tabular}{|c|c|c|c|c|c|}
\hline Variáveis sociodemográficas e familiares & \multicolumn{2}{|c|}{$\begin{array}{c}\text { Não expostas } \\
\quad(n=192)\end{array}$} & \multicolumn{2}{|c|}{$\begin{array}{l}\text { Expostas } \\
(n=191)\end{array}$} & $p$ \\
\hline Faixa etária & \multicolumn{2}{|c|}{$n=191$} & \multicolumn{2}{|c|}{$n=190$} & \multirow{6}{*}{0,054} \\
\hline$<20$ anos & 5 & $(2,6 \%)$ & 4 & $(2,1 \%)$ & \\
\hline $20-29$ anos & 91 & $(47,6 \%)$ & 117 & $(61,6 \%)$ & \\
\hline $30-39$ anos & 68 & $(35,6 \%)$ & 55 & $(28,9 \%)$ & \\
\hline $40-49$ anos & 23 & $(12,0 \%)$ & 13 & $(6,8 \%)$ & \\
\hline 50 anos ou mais & 4 & $(2,1 \%)$ & 1 & $(0,5 \%)$ & \\
\hline Estado civil & \multicolumn{2}{|c|}{$\mathrm{n}=192$} & \multicolumn{2}{|c|}{$\mathrm{n}=191$} & \multirow{5}{*}{$0,036^{*}$} \\
\hline Solteira & 75 & $(39,1 \%)$ & 95 & $(49,7 \%)$ & \\
\hline Casada ou com companheiro & 86 & $(44,8 \%)$ & 81 & $(42,4 \%)$ & \\
\hline Separada ou divorciada & 20 & $(10,4 \%)$ & 8 & $(4,2 \%)$ & \\
\hline Viúva & 11 & $(5,7 \%)$ & 7 & $(3,7 \%)$ & \\
\hline Escolaridade & \multicolumn{2}{|c|}{$\mathrm{n}=186$} & \multicolumn{2}{|c|}{$\mathrm{n}=187$} & \multirow{6}{*}{$<0,001^{*}$} \\
\hline Alfabetizada & 1 & $(0,5 \%)$ & 2 & $(1,1 \%)$ & \\
\hline Completou até $4^{a}$ série do Ensino Fundamental & 12 & $(6,5 \%)$ & 6 & $(3,2 \%)$ & \\
\hline Completou até $8^{\underline{a}}$ série do Ensino Fundamental & 55 & $(29,6 \%)$ & 23 & $(12,3 \%)$ & \\
\hline Completou até $3^{3}$ série do Ensino Médio & 108 & $(58,1 \%)$ & 133 & $(71,1 \%)$ & \\
\hline Completou Nível superior & 10 & $(5,4 \%)$ & 23 & $(12,3 \%)$ & \\
\hline
\end{tabular}

* Significante 
Tabela 2 Aspectos relacionados ao trabalho com substâncias químicas entre trabalhadoras não expostas e expostas ao cianeto na indústria de joias folheadas, Limeira, 2007

\begin{tabular}{|c|c|c|c|c|c|}
\hline Aspectos relacionados ao trabalho com substâncias químicas & \multicolumn{2}{|c|}{$\begin{array}{l}\text { Não expostas } \\
(n=192)\end{array}$} & \multicolumn{2}{|c|}{$\begin{array}{l}\text { Expostas } \\
(n=191)\end{array}$} & $p$ \\
\hline $\begin{array}{l}\text { Trabalha com outras substâncias / compostos químicos } \\
\text { associados }\end{array}$ & \multicolumn{2}{|c|}{$\mathrm{n}=175$} & \multicolumn{2}{|c|}{$\mathrm{n}=188$} & \multirow{4}{*}{$<0,001^{*}$} \\
\hline Sim & 9 & $(5,1 \%)$ & 103 & $(54,8 \%)$ & \\
\hline Não & 162 & $(92,6 \%)$ & 79 & $(42,0 \%)$ & \\
\hline Não sabe & 4 & $(2,3 \%)$ & 6 & $(3,2 \%)$ & \\
\hline Com quais tipos de substâncias químicas trabalha & \multicolumn{2}{|c|}{$\mathrm{n}=191$} & \multicolumn{2}{|c|}{$\mathrm{n}=185$} & \\
\hline Não trabalha / não sabe se trabalha / sem resposta & 183 & $(95,8 \%)$ & 88 & $(47,6 \%)$ & \\
\hline Ácido & 1 & $(0,5 \%)$ & 0 & $(0,0 \%)$ & \\
\hline Ácido para solda fria & 1 & $(0,5 \%)$ & 0 & $(0,0 \%)$ & \\
\hline Ácido, amoníaco & 1 & $(0,5 \%)$ & 0 & $(0,0 \%)$ & \\
\hline Cianetos & 0 & $(0,0 \%)$ & 78 & $(42,2 \%)$ & \\
\hline Cianeto associado a outros químicos ${ }^{\text {桠 }}$ & 2 & $(1,0 \%)$ & 13 & $(6,7 \%)$ & \\
\hline Produto usado na solda fria & 1 & $(0,5 \%)$ & 0 & $(0,0 \%)$ & \\
\hline Produtos de limpeza & 1 & $(0,5 \%)$ & 0 & $(0,0 \%)$ & \\
\hline Outros produtos & 1 & $(0,5 \%)$ & 6 & $(3,4 \%)$ & \\
\hline Por quantas horas trabalha com esses compostos & \multicolumn{2}{|c|}{$\mathrm{n}=191$} & \multicolumn{2}{|c|}{$\mathrm{n}=184$} & \\
\hline Não trabalha / não sabe se trabalha / sem resposta & 183 & $(95,8 \%)$ & 88 & $(47,6 \%)$ & \\
\hline Até 4 horas/dia & 2 & $(1,0 \%)$ & 16 & $(8,7 \%)$ & \\
\hline De 4 até 8 horas/dia & 3 & $(1,6 \%)$ & 69 & $(37,5 \%)$ & \\
\hline Mais de 8 horas/dia & 3 & $(1,6 \%)$ & 11 & $(6,0 \%)$ & \\
\hline
\end{tabular}

\footnotetext{
"Significante

***: Cianeto associado a outros químicos inclui produtos/substâncias relatados pelas trabalhadoras: ácido sulfúrico, fosfato, ácidos nítricos e decapante, outros ácidos/ácidos diversos, liga 88, estanho, níquel, chumbo, cobre.
}

A diferença entre os grupos quanto à distribuição do uso de equipamento de proteção individual no trabalho foi estatisticamente significante ( $p<0,001)$, sendo que o grupo exposto apresentou mais mulheres que usam equipamento $(57,8 \%)$ e usam às vezes $(10,7 \%)$ e menor quantidade de mulheres que não usam $(31,6 \%)$ quando comparadas às do grupo não exposto $(8,5 \%$, $2,7 \%$ e $88,8 \%$ respectivamente). O fornecimento de equipamentos de segurança pela empresa foi estatisticamente significante $(\mathrm{p}<0,001)$, sendo que o grupo exposto apresentou maior proporção de mulheres que recebem equipamento de segurança da empresa $(96,8 \%)$ do que o grupo não exposto $(19,9 \%)$.

As trabalhadoras expostas ao cianeto afirmaram a utilização de: luvas $(85,86 \%)$, máscara $(67,02 \%)$, botas e/ou sapatos $(41,36 \%)$, óculos $(39,27 \%)$, avental, jaleco e/ou capa $(10,47 \%)$, protetor auricular $(3,14 \%)$, uniforme $(3,66 \%)$, capacete $(3,66 \%)$ e toca $(0,52 \%)$, sendo que uma $(0,52 \%)$ disse usar tudo, uma $(0,52 \%)$ não sabe quais equipamentos usa e $7,33 \%$ não responderam essa questão. Já o grupo não exposto ao cianeto relatou a utilização de luvas $(14,06 \%)$, máscara $(7,81 \%)$, botas/sapatos $(6,25 \%)$, protetor auricular $(6,25 \%)$, avental e/ou capas $(3,13 \%)$, uniforme (2,08\%), óculos ( $0,52 \%)$, outros equipamentos $(1,04 \%)$, sendo que $81,77 \%$ não responderam e uma $(0,52 \%)$ não soube dizer quais equipamentos usa.

Foi encontrada diferença estatisticamente significante entre os grupos quanto à distribuição da presença de lesões por acidentes ou doenças, confirmadas por alguma avaliação médica anterior $(p=0,042)$, sendo que o grupo exposto apresentou menor proporção de mulheres com lesões ou doenças $(35,6 \%)$ do que o grupo não exposto (45,8\%). Também houve diferença entre os grupos quanto à distribuição do número de lesões por acidentes ou doenças $(p=0,039)$, sendo que o grupo exposto apresentou um número médio de doenças e lesões de 1,96 (dp $=0,88)$ menor que o grupo não exposto, de 1,89 (dp = 1,12).

Com relação às morbidades referidas através da aplicação do ICT, o grupo não exposto apresentou maior proporção de mulheres com lesão nas costas (7,3\%), nos braços e/ou mãos $(16,1 \%)$, nas pernas e/ ou pés $(5,2 \%)$ e com gastrite ou irritação duodenal $(5,2 \%)$. Já o grupo exposto ao cianeto apresentou maior proporção de mulheres com bronquite crônica $(9,9 \%)$, sinusite crônica (11\%) e com alguma doença de tireoide ou bócio (11\%) (Tabela 3).

Quanto ao hábito de fumar, encontrou-se diferença entre os grupos quanto à distribuição do tempo de tabagismo $(\mathrm{p}=0,018)$, com o grupo exposto ao cianeto apresentando 8 anos $(\mathrm{dp}=4,7)$ e o grupo não exposto, 13,1 anos $(\mathrm{dp}=7,3)$. Não foi encontrada diferença estatisticamente significante entre os grupos quanto à distribuição da classificação da escala Fagerström ( $p$ = $0,660)$, quanto à distribuição dos hábitos de fumar ( $p$ $=0,138)$, nem quanto à distribuição do número de cigarros fumados por dia $(p=0,837)$, o que descartou a possibilidade de que os relatos de bronquite e sinusite estivessem associados ao hábito de fumar (Tabela 4). 
Tabela 3 Lesões e doenças com diagnóstico médico entre trabalhadoras não expostas e expostas ao cianeto na indústria de joias folheadas, Limeira, 2007

\begin{tabular}{|c|c|c|c|c|c|}
\hline \multirow{2}{*}{$\begin{array}{l}\text { Lesões por acidentes ou doenças }-n(\%) \\
\text { 01. Lesão nas costas }\end{array}$} & \multicolumn{2}{|c|}{$\begin{array}{c}\text { Não Expostas } \\
(n=192)\end{array}$} & \multicolumn{2}{|c|}{$\begin{array}{l}\text { Expostas } \\
(n=191)\end{array}$} & \multirow{2}{*}{$\frac{p}{0,007^{*}}$} \\
\hline & 14 & $(7,3 \%)$ & 3 & $(1,6 \%)$ & \\
\hline 02. Lesão nos braços / mãos & 31 & $(16,1 \%)$ & 4 & $(2,1 \%)$ & $<0,001 *$ \\
\hline 03. Lesão nas pernas / pés & 10 & $(5,2 \%)$ & 2 & $(1,0 \%)$ & $0,019 *$ \\
\hline 04. Lesão em outras partes do corpo & 0 & $(0,0 \%)$ & 3 & $(1,6 \%)$ & 0,123 \\
\hline $\begin{array}{l}\text { 05. Doença na parte superior das costas ou região do pescoço, } \\
\text { com dores frequentes }\end{array}$ & 2 & $(1,0 \%)$ & 3 & $(1,6 \%)$ & 0,685 \\
\hline 06. Doença na parte inferior das costas com dores frequentes & 1 & $(0,5 \%)$ & 3 & $(1,6 \%)$ & 0,372 \\
\hline 07. Dor nas costas que se irradia para a perna (ciática) & 5 & $(2,6 \%)$ & 4 & $(2,1 \%)$ & 0,100 \\
\hline $\begin{array}{l}\text { 08. Doença musculoesquelética afetando os membros (braços e } \\
\text { pernas) com dores frequentes }\end{array}$ & 2 & $(1,0 \%)$ & 1 & $(0,5 \%)$ & 0,100 \\
\hline 09. Artrite reumatoide & 5 & $(2,6 \%)$ & 1 & $(0,5 \%)$ & 0,215 \\
\hline 10. Outra doença musculoesquelética & 0 & $(0,0 \%)$ & 1 & $(0,5 \%)$ & 0,499 \\
\hline 11. Hipertensão arterial & 20 & $(10,4 \%)$ & 12 & $(6,3 \%)$ & 0,144 \\
\hline 12. Doença coronariana, dor no peito durante exercício & 0 & $(0,0 \%)$ & 1 & $(0,5 \%)$ & 0,499 \\
\hline 13. Infarto do miocárdio, trombose coronariana & 0 & $(0,0 \%)$ & 0 & $(0,0 \%)$ & --- \\
\hline 14. Insuficiência cardíaca & 1 & $(0,5 \%)$ & 0 & $(0,0 \%)$ & 0,100 \\
\hline 15. Outra doença cardiovascular & 0 & $(0,0 \%)$ & 1 & $(0,5 \%)$ & 0,499 \\
\hline 16. Infecções repetidas no trato respiratório & 2 & $(1,0 \%)$ & 3 & $(1,6 \%)$ & 0,685 \\
\hline 17. Bronquite crônica & 8 & $(4,2 \%)$ & 19 & $(9,9 \%)$ & 0,027 * \\
\hline 18. Sinusite crônica & 9 & $(4,7 \%)$ & 21 & $(11,0 \%)$ & $0,022 \%$ \\
\hline 19. Asma & 5 & $(2,6 \%)$ & 6 & $(3,1 \%)$ & 0,753 \\
\hline 20. Enfisema & 4 & $(2,1 \%)$ & 0 & $(0,0 \%)$ & 0,123 \\
\hline 21. Tuberculose pulmonar & 0 & $(0,0 \%)$ & 0 & $(0,0 \%)$ & --- \\
\hline 22. Outra doença respiratória & 1 & $(0,5 \%)$ & 6 & $(3,1 \%)$ & 0,067 \\
\hline 23. Distúrbio emocional severo & 3 & $(1,6 \%)$ & 0 & $(0,0 \%)$ & 0,248 \\
\hline 24. Distúrbio emocional leve & 5 & $(2,6 \%)$ & 3 & $(1,6 \%)$ & 0,724 \\
\hline 25. Problema ou diminuição na audição & 6 & $(3,1 \%)$ & 2 & $(1,0 \%)$ & 0,284 \\
\hline 26. Doença ou lesão da visão & 6 & $(3,1 \%)$ & 4 & $(2,1 \%)$ & 0,751 \\
\hline 27. Doença neurológica & 1 & $(0,5 \%)$ & 1 & $(0,5 \%)$ & 0,100 \\
\hline 28. Outra doença neurológica ou dos órgãos dos sentidos & 0 & $(0,0 \%)$ & 1 & $(0,5 \%)$ & 0,499 \\
\hline 29. Pedras ou doença da vesícula biliar & 1 & $(0,5 \%)$ & 2 & $(1,0 \%)$ & 0,623 \\
\hline 30. Doenças do pâncreas ou do fígado & 0 & $(0,0 \%)$ & 1 & $(0,5 \%)$ & 0,499 \\
\hline 31. Úlcera gástrica ou duodenal & 3 & $(1,6 \%)$ & 4 & $(2,1 \%)$ & 0,724 \\
\hline 32. Gastrite ou irritação duodenal & 10 & $(5,2 \%)$ & 3 & $(1,6 \%)$ & $0,049 *$ \\
\hline 33. Colite ou irritação do cólon & 1 & $(0,5 \%)$ & 1 & $(0,5 \%)$ & 1,000 \\
\hline 34. Outra doença digestiva & 0 & $(0,0 \%)$ & 1 & $(0,5 \%)$ & 0,499 \\
\hline 35. Infecção das vias urinárias & 8 & $(4,2 \%)$ & 2 & $(1,0 \%)$ & 0,105 \\
\hline 36. Doença dos rins & 1 & $(0,5 \%)$ & 1 & $(0,5 \%)$ & 0,100 \\
\hline 37. Doença nos genitais e aparelho reprodutor & 3 & $(1,6 \%)$ & 3 & $(1,6 \%)$ & 0,100 \\
\hline 38. Outra doença geniturinária & 1 & $(0,5 \%)$ & 1 & $(0,5 \%)$ & 0,110 \\
\hline 39. Alergia, eczema & 10 & $(5,2 \%)$ & 8 & $(4,2 \%)$ & 0,637 \\
\hline 40. Outra erupção & 0 & $(0,0 \%)$ & 0 & $(0,0 \%)$ & --- \\
\hline 41. Outra doença de pele & 1 & $(0,5 \%)$ & 0 & $(0,0 \%)$ & 0,100 \\
\hline 42. Tumor benigno & 0 & $(0,0 \%)$ & 0 & $(0,0 \%)$ & -- \\
\hline 43. Tumor maligno (câncer) & 0 & $(0,0 \%)$ & 0 & $(0,0 \%)$ & --- \\
\hline 44. Obesidade & 7 & $(3,6 \%)$ & 9 & $(4,7 \%)$ & 0,602 \\
\hline 45. Diabetes & 7 & $(3,6 \%)$ & 3 & $(1,6 \%)$ & 0,337 \\
\hline 46. Bócio ou outra doença de tireoide & 9 & $(4,7 \%)$ & 21 & $(11,0 \%)$ & $0,022 *$ \\
\hline 47. Outra doença endócrina ou metabólica & 1 & $(0,5 \%)$ & 0 & $(0,0 \%)$ & 0,100 \\
\hline 48. Anemia & 11 & $(5,7 \%)$ & 4 & $(2,1 \%)$ & 0,067 \\
\hline 49. Outra doença do sangue & 1 & $(0,5 \%)$ & 0 & $(0,0 \%)$ & 0,100 \\
\hline 50. Defeito de nascimento & 0 & $(0,0 \%)$ & 0 & $(0,0 \%)$ & --- \\
\hline 51. Outro problema ou doença & 0 & $(0,0 \%)$ & 0 & $(0,0 \%)$ & --- \\
\hline
\end{tabular}

"Significante 
Tabela 4 Variáveis relacionadas ao hábito de fumar entre as trabalhadoras não expostas e expostas ao cianeto na indústria de joias folheadas, Limeira, 2007

\begin{tabular}{|c|c|c|c|}
\hline Variáveis relacionadas ao hábito de fumar & $\begin{array}{c}\text { Não expostas } \\
(n=192)\end{array}$ & $\begin{array}{l}\text { Expostas } \\
(n=191)\end{array}$ & $p$ \\
\hline Fuma & $\mathrm{n}=188$ & $n=191$ & \\
\hline não, nunca fumou & $(66,0 \%)$ & $(66,5 \%)$ & \\
\hline não, mas já fumou & $(17,6 \%)$ & $(23,0 \%)$ & 0,138 \\
\hline Sim & $(16,5 \%)$ & $(10,5 \%)$ & \\
\hline Fagerström: classificação & $\mathrm{n}=31$ & $\mathrm{n}=20$ & \\
\hline Dependência leve ( 0 a 4 ) & $(54,8 \%)$ & $(50,0 \%)$ & \\
\hline Dependência moderada (5 a 7) & $(29,0 \%)$ & $(40,0 \%)$ & 0,660 \\
\hline Dependência alta (8 a 10) & $5 \quad(16,1 \%)$ & $2 \quad(10,0 \%)$ & \\
\hline Número de cigarros fumados por dia & $\mathrm{n}=31$ & $\mathrm{n}=17$ & \\
\hline Média $\pm d p$ & $16,1 \pm 8,0$ & $15,2 \pm 8,4$ & \\
\hline Mediana & 15 & 16 & 0,837 \\
\hline Mínimo - máximo & $4-30$ & $3-30$ & \\
\hline Há quantos anos é fumante & $\mathrm{n}=29$ & $\mathrm{n}=16$ & \\
\hline Média $\pm \mathrm{dp}$ & $13,1 \pm 7,3$ & $8,0 \pm 4,7$ & \\
\hline Mediana & 10 & 7,5 & 0,018 * \\
\hline Mínimo - máximo & $3-30$ & $2-20$ & \\
\hline Escala de Tolerância de Fagerström & $\mathrm{n}=31$ & $\mathrm{n}=20$ & \\
\hline Média $\pm \mathrm{dp}$ & $4,19 \pm 2,8$ & $3,40 \pm 3,1$ & \\
\hline Mediana & 4 & 4,5 & 0,288 \\
\hline Mínimo - máximo & $0-9$ & $0-9$ & \\
\hline
\end{tabular}

* significante

\section{Discussão}

Este estudo verificou a existência de dois perfis de adoecimento, ou seja, estimou diferentes prevalências para as morbidades autorreferidas, as quais se mostraram correspondentes às condições e à organização do trabalho neste setor produtivo. Enquanto as trabalhadoras que realizavam atividades monótonas e repetitivas referiram lesão em braços, mãos, pernas, pés e costas - o que possibilitou a visualização da hipersolicitação do corpo na realização do trabalho de montagem -, as disfunções tireoideanas, a bronquite e a sinusite crônicas foram as morbidades mais referidas pelas mulheres que estavam no grupo de galvanoplastas, consideradas expostas ao cianeto.

Pelo fato da autoavaliação do estado de saúde se constituir em um dado facilmente coletado, com índices de $80 \%$ de concordância entre a autoavaliação do estado de saúde e a avaliação clínica da presença ou ausência de condição crônica (BARROS et al, 2009), este foi o método empregado para estimar a prevalên- cia de agravos e doenças em mulheres trabalhadoras na indústria de bijuterias e folheados em Limeira.

No que tange ao uso da autoavaliação para a estimativa de LER/DORT, Poletto et al. (2007) afirmam que existe uma relação clara entre o relato de sintomas e a presença de sinais desses distúrbios identificados no exame clínico. Assim, a diferença estatisticamente significativa observada na prevalência de lesões nas mãos e nos braços ( $\mathrm{p}<0,001$ ), nas costas $(p=0,007)$ e nas pernas e nos pés $(p=0,019)$ podem se transformar em incapacidade temporária ou permanente de jovens.

Com relação às morbidades relatadas pelo grupo exposto ao cianeto, houve maior prevalência de agravos no trato respiratório, como a bronquite e a sinusite crônicas, os quais não foram associados ao hábito de fumar, que poderia ter agido como aditivo.

O principal metabólito do cianeto, o tiocianato, exerce uma ação sobre a tireoide e é um composto bociogênico que altera a síntese dos hormônios tireoideanos (WORLD HEALTH OR- 
GANIZATION, 2007). Ao ser excretado pela urina, o tiocianato é capaz de indicar uma exposição ambiental. A maior estabilidade do tiocianato (compa rado ao cianeto) o torna mais adequado para fins de biomonitoramento, contanto que se atente para exposições extraocupacionais, como tabagismo e alimentação (WORLD HEALTH ORGANIZATION, 2007; SANTOS et al., 1990). Isto poderá ser verificado em estudos de coorte futuros que verifiquem a associação de fatores relacionados a exposições laborais e extralaborais com sintomatologias e doenças. Também é importante colocar que outras síndromes, como o cretinismo endêmico, têm sido associadas à intoxicação crônica do cianeto, a falhas no mecanismo de desintoxicação, a deficiências nutricionais ou à combinação desses fatores (AMERICAN CONFERENCE OF GOVERNMENTAL INDUSTRIAL HYGIENISTS, 1986) e devem ser melhor investigadas. Neste estudo, não foi possível a realização de exames laboratoriais para verificar a exposição ao cianeto por conta do alto custo.

Devido ao alto custo dos exames clínico-laboratorais, o indicador de morbidade referida se mostrou uma alternativa que forneceu dados para o mapeamento das condições gerais de saúde percebidas pelas próprias trabalhadoras.

O uso desse mapeamento se baseia no que afirmam Ferraro e Su (2000), segundo os quais tanto os exames clínicos, como a autoavaliação de saúde são medidas válidas na predição de futuras incapacidades, principalmente para doenças crônicas não severas, e Lima-Costa et al. (2007), que verificaram que as doenças que exigem acompanhamento médico e laboratorial tendem a ser melhor informadas pelos entrevistados, pois depende da percepção de sinais e sintomas da doença e do acesso e da qualidade dos serviços médico-diagnósticos.

Mesmo que a autoavaliação em saúde seja influenciada pelo acesso aos serviços de saúde (e possível diagnóstico da doença) e pelo viés de memória, estudos mostram a validade de informações referidas com sensibilidade e especificidade para detectar condições relacionadas à saúde (LIMA-COSTA; PEIXOTO; FIRMO, 2004; QUESNEL-VALLÉE, 2007). Azevedo et al. (2006) afirmam que há maior concordância entre informações de inquérito e de registros médicos em casos de doenças que provocam maior grau de incapacidade, sendo que, em casos de doenças crônicas, em que o paciente só reconhece o problema após o diagnóstico médico, os inquéritos tendem a subestimar sua prevalência, o que demonstra a necessidade de outros instrumentos para determinar a prevalência dos agravos que atingem as trabalhadoras de Limeira.
De qualquer forma, o estudo usando morbidade referida permite avaliar o estado de saúde da população, registrar o número de pessoas incapacitadas ou funcionalmente doentes, avaliar o uso de diferentes tipos de serviços de saúde, além de obter o que o autor chama de "marco zero" para planejamento em saúde (CÉSAR et al., 1996). Ainda, para Almeida et al. (2002), a morbidade referida é um indicador que possibilita levantar as demandas por serviços, conhecer as condições da população estudada, avaliar as políticas de saúde e auxiliar em novas propostas em saúde pública. Assim, a presença de um problema crônico de saúde, seja o seu conhecimento pelo indivíduo resultante de um diagnóstico médico anterior ou de uma autoavaliação, constitui um dos determinantes proximais mais fortes para a procura e a utilização de serviços de saúde.

No Brasil, poucos trabalhos abordam a epidemiologia da sinusite, a qual foi referida por $11 \%$ das trabalhadoras, prevalência maior do que a encontrada em estudo similar que observou a prevalência de 7,3\% em enfermeiras (VASCONCELOS, 2009), o que levanta o questionamento sobre a causa ou o agravamento da sinusite por substâncias químicas irritantes, tanto na forma de gases e vapores, quanto de névoas e poeiras (DIAS, 2001). Este fator deve ser considerado visto que nos Estados Unidos a sinusite crônica representa uma das principais causas de absenteísmo no trabalho e na escola, atingindo 14\% da população (CAUWENBERGE; WATELET, 2000; BENNINGER et al., 2003). No Canadá, verificou-se uma prevalência de $5 \%$ na população geral, sendo mais comum em mulheres adultas (CHEAN; DALES; LIN, 2003).

A bronquite crônica, referida por $9 \%$ das trabalhadoras expostas ao cianeto, foi outro agravo do trato respiratório relatado. No Brasil, em estudos populacionais, Vasconcelos (2009) verificou uma prevalência de $0,4 \%$ de bronquite através da aplicação do ICT, e a PNAD de 2003, uma prevalência de asma ou bronquite crônica autorreferida de 2,63\% no nordeste e de 5,89\% no sul do Brasil (BERNAT et al., 2009). Entretanto, em suinocultores, foi verificada a prevalência de $5,1 \%$ de bronquite crônica através de questionário aplicado (COSTA; TEIXEIRA; FREITAS, 2007). De acordo com Algranti (2001), alguns grupos ocupacionais estudados apresentam prevalências elevadas de bronquite crônica diagnosticadas através de questionários de sintomas respiratórios, como os trabalhadores inseridos em minas de carvão $(27,9 \%)$, na fiação de linho (15\%), em silos de grãos (23,2\%) e ex-trabalhadores da extração de cimento-amianto (16,7\%). Entretanto, diferentemente do que foi encontrado em nosso estudo, o tabagismo se mostrou uma variável significativa para a maior prevalência junto aos grupos dos mineiros e dos trabalhadores dos silos. Já nos ex-trabalhadores do cimento-amianto, a exposição ocupacional associou-se 
significativamente à maior prevalência de bronquite crônica, demonstrando que estudos populacionais podem revelar sintomas respiratórios crônicos associados à exposição a poeiras, gases e fumos.

O grupo de galvanoplastas também relatou uma maior prevalência de disfunções tireoidianas e/ou hipotireoidismo (11\%), apresentando uma diferença estatisticamente significante quando comparado ao grupo não exposto ao cianeto $(\mathrm{p}=0,022)$. De acordo com a literatura, a frequência do hipotireoidismo pode variar consideravelmente conforme a população estudada, sendo sua prevalência de $0,6 \%$ (CANARIS et al., 2000) a 3,7\% nos Estados Unidos (AOKI, 2007), de 1\% no Chile (FARDELLA et al., 2001) e de $4 \%$ em mulheres com mais de 60 anos (GUSSEKLOO et al., 2004). Já em trabalhadoras rurais expostas a organoclarados, pesticidas e herbicidas, foi encontrada uma prevalência de hipotireoidismo de 12,5\% (WHITNEY et al., 2010), próxima à encontrada no estudo com as trabalhadoras expostas ao cianeto. Em estudo realizado no Brasil, Vasconcelos (2009), também utilizando a autoavaliação, verificou a prevalência de 1,8\% de problemas relacionados à tireoide junto a enfermeiras.

Por ser transversal, esta pesquisa apresentou limitações por não permitir o estabelecimento de relações causais, o que pode ser retomado em outros desenhos de pesquisa envolvendo o segmento de joias e bijuterias. Além disso, não foi realizada a avaliação da exposição, mas o fato de apenas 54,8\% das mulheres do grupo exposto relatar contato direto com produtos e substâncias químicas não descarta a exposição das outras trabalhadoras ao cianeto, visto que a produção é organizada no mesmo ambiente ou galpão, e mesmo as trabalhadoras que não fazem o banho da peça com cianeto se encontram expostas à substância. E apesar de apenas $37,5 \%$ terem relatado uma exposição cotidiana de 4 a 8 horas/dia, estudos detalhados sobre a exposição devem ser realizados de modo a verificar o tempo e a intensidade da exposição de trabalhadoras que lidam direta ou indiretamente com o cianeto e a sua adesão ao uso de Equipamentos de Proteção Individual - o uso de respirador com filtro apropriado é recomendado, além de luvas e roupas de proteção, uma vez que a substância é absorvida facilmente pela via dérmica, tanto na forma líquida, como na gasosa (NIELSEN; OVREBO, 2008). Foi encontrada maior proporção de mulheres com mais anos de estudo no grupo considerado exposto, o que pode ser mais detalhadamente investigado, pois pode indicar maior dificuldade de inserção no mercado de trabalho e aceitação de condições de trabalho precárias frente ao desemprego.

Nesse contexto, a Vigilância em Saúde do Trabalhador, órgão responsável por formular alternativas promotoras de saúde junto às mulheres inseridas nessa cadeia produtiva, é pouco presente nas empresas desta região, sendo praticamente inexistente o monitoramento do manejo adequado dos produtos químicos. O escasso conhecimento acerca do trabalho e das condições de saúde nesse ramo industrial também requer que se estabeleça um diálogo maior entre várias áreas de conhecimento em busca de estabelecer relações causais entre a exposição ao cianeto, outros compostos químicos e agravos referidos. Para isso, deve-se recorrer a testes de laboratório, medições ambientais e biológicas da exposição, avaliações de risco, avaliação neuropsicológica (por entrevista clínica, testes neurológicos e de personalidade padronizados), avaliação de neuropatias derivadas de lesão cerebral e avaliação da neuropatia periférica acerca da condução nervosa. Entende-se, portanto, que essas avaliações são essenciais para a melhor compreensão da relação entre queixas e sintomas de adoecimento e o ambiente de trabalho, bem como para se propor medidas de promoção à saúde no grupo ocupacional estudado.

\section{Agradecimento}

Agradeço ao Professor Angelo Zanaga Trape pelas valiosas orientações durante a pesquisa de doutorado.

\section{Referências}

AGENCY FOR TOXIC SUBSTANCES AND DISEASE REGISTRY. Toxicological profile for cyanide. US Department of Health and Human Services, 2006.

ALGRANTI, E. Tabagismo e ocupação: elo de exposições pouco explorado como estratégia de combate ao tabagismo. Jornal de Pneumologia, v. 27, n. 4, p. VII-VIII, jul./ago. 2001.
ALMEIDA, M. F. et al. Prevalência de doenças crônicas auto-referidas e utilização de serviços de saúde, PNAD/1998, Brasil. Ciência \& Saúde Coletiva, Rio de Janeiro, v. 7, n. 4, p. 743-756, 2002.

AMERICAN CONFERENCE OF GOVERNMENTAL INDUSTRIAL HYGIENISTS. Documentation of the 
threshold limits values and biological exposure indices. 5. ed. Cincinnati: ACGIH, 1986.

AOKI, Y. et al. Serum TSH and total T4 in the United States population and their association with participant characteristics: National Health and Nutrition Examination Survey (NHANES 1999-2002). Thyroid, New York, v. 17, n. 12, p. 1211-1223, 2007.

AZEVEDO, M. B. de et al. Desigualdades sociais na prevalência de doenças crônicas no Brasil, PNAD2003. Ciência \& Saúde Coletiva, Rio de Janeiro, v. 11, n. 4, p. 922-926, out./dez. 2006.

BARROS, M. B. de A. et al. Auto-avaliação da saúde e fatores associados, Brasil, 2006. Revista de Saúde Pública, São Paulo, v. 43, supl. 2, p. 27-37, nov. 2009.

BENNINGER, M. S.; et al. Adult chronic rhinosinusitis: definitions, diagnosis, epidemiology, and pathophysiology. Otolaryngology, Head and Neck Surgery, Rochester, v. 129, n. 3 (Suppl), p. S1-S32, 2003.

BERNAT, A. C. et al. Prevalência de sintomas respiratórios e fatores associados: estudo de base populacional em adultos de Lages, Santa Catarina, Brasil. Cadernos de Saúde Pública, Rio de Janeiro, v. 25, n. 9, p. 1907-1916, 2009.

CANARIS, G. J. et al. The Colorado Thyroid Disease Prevalence Study. Archives of Internal Medicine, Chicago, v. 160, p. 526-534, 2000.

CAUWENBERGE, P. V.; WATELET, J. B. Epidemiology of chronic rhinosinusitis. Thorax, London, v. 55, Suppl. 2, p. S20-S21, 2000.

CESAR, L. G. C. et al. Morbidade referida e utilização de serviços de saúde em localidades urbanas brasileiras: metodologia. Revista de Saúde Pública, São Paulo, v. 30, n. 2, p. 153-160, 1996.

CHEAN, Y.; DALES, R.; LIN, M. The epidemiology of chronic rhinosinusitis in Canadians. Laryngoscope, Saint Louis, v. 113, p. 1199-1205, 2003.

COSTA, M.; TEIXEIRA, P. J. Z.; FREITAS, P. F.

Manifestações respiratórias e doenças de vias aéreas: prevalência e fatores de risco em suinocultores de Braço do Norte, Santa Catarina. Jornal Brasileiro de Pneumologia, Brasília, v. 33, n. 4, p. 380-388, 2007.

CRUZ, M. Avaliação econômica do mercado de jóias e folheados a ouro do município de Juazeiro do Norte. 2001. 50 f. Monografia (Trabalho de Conclusão de Curso em Economia)-Universidade Regional do Cariri, Crato, Ceará, 2001.

CURRY, S. C. Hydrogen cyanide and inorganic cyanide salts. In: SULLIVAN JUNIOR, J. B.; KRIEGER, G. R. Hazardous materials toxicology, clinical principles of environmental health. Baltimore: Williams \& Wilkins, 1992. p. 698-710.

DI FILIPPO, M. et al. Parkinsonism and cognitive impairment following chronic exposure to potassium cyanide. Movement Disorders, New York, v. 23, n. 3, p. 468-470, fev. 2008.

DIAS, E. C. (Org.). Doenças relacionadas ao trabalho: manual de procedimentos para os serviços de saúde. Brasília: Ministério da Saúde do Brasil/OPAS, 2001.

FARDELLA, B. C. et al. Alta prevalencia de enfermedad tiroidea subclínica em sujetos que concurren a control de salud. Revista Medica de Chile, Santiago de Chile, v. 129, n. 2, p. 155-160, fev. 2001.

FERNANDES, L S. C. Arranjo produtivo de jóias e folheados de Juazeiro do Norte: uma proposta que vale ouro. Fortaleza. 2005. 45 f. Monografia (Trabalho de Conclusão de Curso em Ciências Econômicas)Universidade Federal do Ceará, Ceará, 2005.

FERRARO, K. F.; SU, Y. Physician-evaluated and selfreported morbidity for predicting disability. American Journal of Public Health, Boston, v. 90, n. 1, p. 103-108, 2000.

FIGUEIREDO, V. C. N. Trabalho e gênero: condições de saúde das mulheres no setor de bijuterias e folheados. 2008. 201 f. Tese (Doutorado em Saúde Coletiva)Faculdade de Ciências Médicas, Unicamp. Campinas, 2008.

FREITAS, M. C.; LIMA, L. H. C. Diagnóstico e tratamento do hipotireoidismo. In: VILAR, L.; CASTELLAR, E.; MOURA, E. Endocrinologia clínica. Rio de Janeiro: Medsi, 1999. p. 134-144.

GOMES, K. R. O.; TANAKA, A. C. d'A. Morbidade referida e uso dos serviços de saúde por mulheres trabalhadoras, Município de São Paulo. Revista de Saúde Pública, São Paulo, v. 37, n. 1, p. 75-82, 2003.

GRANT, W. M. Toxicology of the Eye. Springfield: Charles C. Thomas, 1974.

GUSSEKLOO, J. et al. Thyroid status, disability and cognitive function, and survival in old age. Journal of the American Medical Association, Chicago, v. 292, p. 2591-2599, 2004.

HALL, A. H.; RUMACK, B. H. Cyanide and related compounds. In: HADDAD, R. M.; SHANNON, M. W.; WINCHESTER, J. F. Clinical manegement of poisoning and drug overdose. 3. ed. Philadelfia: W. B. Saunders, 1998. p. 899-905.

HALTY, L. S. et al. Análise da utilização do Questionário de Tolerância de Fargeström (QTF) como instrumento de medida da dependência nicotínica. Jornal de Pneumologia, São Paulo, v. 28, n. 4, p. 180186, 2002.

LEBRÃO, M. L.; DUARTE, Y. A. de O. SABE - Saúde, bem-estar e envelhecimento - O projeto Sabe no município de São Paulo: uma abordagem inicial. Brasília: Organização Pan-Americana da Saúde, 2003.

LEWIS, R. J. Sax's dangerous properties of industrial materials. 9. ed. New York: Van Nostrand Reinhold, 1996. v. 1-3. 
LIMA-COSTA, M. F. et al. Validade do diabetes autoreferido e seus determinantes: evidências do projeto Bambuí. Revista de Saúde Pública, São Paulo, v. 41, n. 6, p. 947-953, dez. 2007.

LIMA-COSTA, M. F.; PEIXOTO, S. V.; FIRMO, J. O. A. Validade da hipertensão arterial auto-referida e seus determinantes (Projeto Bambuí). Revista de Saúde Pública, São Paulo, v. 38, n. 5, p. 637-642, 2004.

MARQUES, A. C. P. R.; RIBEIRO, M. Nicotina: abuso e dependência. In: LARANJEIRA, R. Usuários de substâncias psicoativas: abordagem, diagnóstico e tratamento. São Paulo: Conselho Regional de Medicina do Estado de São Paulo/Associação Médica Brasileira, 2003. p. 49-58.

MELO, R. A. de; CAMPINAS, L. L. S. L. Multiculturalidade e morbidade referida por imigrantes bolivianos na Estratégia Saúde da Família. Mundo da Saúde, São Paulo, v. 24, n. 1, p. 25-35, 2010.

MONTELIUS, J. (Ed.) Hydrogen cyanide, sodium cyanide and potassium cyanide. Criteria Group for occupational standards national institute for working life S-112 79 Stocholm, Sweden. In: . Scientific basis for swedish occupational stand $\overline{a r d s ~ X X I I . ~}$ Translation: Frances Van Sant. Stockholm: NIWL, 2001. p. 43-59. (Arbete och Hälsa, NR 2001:20).

NIELSEN, G. D.; OVREBO, S. Background, approaches and recent trends for setting health-based occupational exposure limits: a minireview. Regulatory Toxicology and Pharmacology, v. 51, n. 3, p. 253-269, 2008.

PENTORE, R.; VENNERI, A.; NICHELLI, P. Accidental choke-cherry poisoning: early symptoms and neurological sequelae of an unusual case of cyanide intoxication. The Italian Journal of Neurological Sciences, v. 17, n. 3, p. 233-235, 1996.

PINHEIRO, R. S. et al. Gênero, morbidade, acesso e utilização de serviços de saúde no Brasil. Ciência \& Saúde Coletiva, Rio de Janeiro, v. 7, n. 4, p. 687-707, 2002.

POLETTO, P. R. et al. Relação entre o relato clínico e o exame físico na avaliação de distúrbios osteomusculares relacionados ao trabalho. Fisioterapia e Pesquisa, São Paulo, v. 14, n. 1, p. 42-46, 2007.

PONTES, A. N. A. et al. Prevalência de doenças da tireóide em uma comunidade do nordeste brasileiro. Arquivos Brasileiros de Endocrinologia \& Metabolismo, São Paulo, v. 46, n. 5, p. 544-549, 2002.

QUESNEL-VALLÉE, A. Self-rated heatlh: caught in the crossfire of the quest for "true" health? International Journal of Epidemiology, v. 36, n. 6, p. 1161-1164, 2007.
QUIROGA, P.; OLMOS, V. Revisión de la toxicocinética y la toxicodinamia del ácido cianhídrico y los cianuros. Acta Toxicologica Argentina, Buenos Aires, v. 17, n. 1, jan./jul. 2009.

SAMPAIO, S. E. K. Sistemas Locais de Produção: estudo de caso da indústria de jóias e bijuterias em Limeira (SP). 2002. 50 f. Monografia (Iniciação Científica)-Instituto de Economia, Universidade Estadual de Campinas. Campinas, 2002.

SANTOS, M. S.; YAMANAKA, H. T.; PACHECO, C. E. M. Bijuterias. São Paulo: Cetesb, 2005. Disponível em: <http://www.cetesb.sp.gov.br>. Acesso em: 3 jan. 2010 .

SANTOS, U. P. et al. Uso de tiocianato urinário como indicador de exposição a cianetos. Revista Brasileira de Saúde Ocupacional, São Paulo, v. 18, n. 69, p. 7173, 1990.

SILVA, C. S. Um estudo crítico sobre a saúde dos trabalhadores de galvânicas por meio das relações entre as avaliações ambientais, biológicas e otorrinolaringológicas. 1998. 168 f. Tese (Doutorado em Química Analítica)-Instituto de Química: Universidade de São Paulo, São Paulo, 1998.

SUZIGAN, W. et al. Aglomerações industriais no Estado de São Paulo. Economia Aplicada, Ribeirão Preto, v. 5, n. 4, p. 695-717, 2001.

TUOMI, K. et al. Índice de capacidade para o trabalho. São Carlos: EdUFSCar, 2005

VASCONCELOS, S. P. Avaliação da capacidade para o trabalho e fadiga entre trabalhadores de enfermagem em um hospital de urgência e emergência na Amazônia Ocidental. 2009. 100 f. Dissertação (Mestrado em Ciências)-Faculdade de Saúde Pública, Universidade de São Paulo. São Paulo, 2009.

WHITNEY, S. et al. Pesticide use and thyroid disease among women in Agriculture Health Study. American Journal of Epidemiology, Baltimore, v. 171, n. 4, p. 455464, 2010.

WORLD HEALTH ORGANIZATION. Cyanide in drinking-water - Background document for development of WHO Guidelines for Drinkingwater quality. Geneva: World Health Organization, 2007. Disponível em: < http://www.who.int/water sanitation health/dwq/chemicals/second addendūm cyanide_short_term\%20_4_.pdf >. Acesso em: $15 \mathrm{fev}$. 2009.

ZHANG, X. et al. Cyanide-induced death of dopaminergic cells is mediated by uncoupling protein-2 up-regulation and reduced Bcl-2 expression. Toxicology and Applied Pharmacology, v. 238, p. 11-19, 2009 . 